\title{
The role of polymer compatibility in the adhesion between surfaces saturated with modified dextrans
}

\author{
Malin Eriksson ${ }^{\mathrm{a}, *}$, Shannon M. Notley ${ }^{\mathrm{b}}$, Robert Pelton ${ }^{\mathrm{c}}$, Lars Wågberg ${ }^{\mathrm{d}}$ \\ a Carmeda AB, Kanalvägen 3B, SE-194 61 Upplands Väsby, Sweden \\ ${ }^{\mathrm{b}}$ Department of Applied Mathematics, Research School of Physical Sciences and Engineering, Australian National University, Canberra 0200 ACT, Australia \\ ${ }^{c}$ McMaster University, Centre for Pulp and Paper Research, Hamilton, ON L8S 4L7, Canada \\ d Department of Fibre and Polymer Technology, School of Chemical Science and Engineering, KTH, Royal Institute of Technology, SE-100 44 Stockholm, Sweden
}

Received 29 November 2006; accepted 21 January 2007

Available online 6 February 2007

\begin{abstract}
Wet and dry adhesion between dextran-coated surfaces were measured aiming to understand the influence of polymer compatibility. The wet adhesion measurements were performed using the atomic force microscope (AFM) colloidal probe technique whereas the dry adhesion measurements were performed using the micro adhesion measurement apparatus (MAMA). Two types of dextrans were used, one cationically modified dextran (DEX) and one that was both cationically and hydrophobically modified (HDEX), leading to three different combinations of polymer-coated surfaces; (1) DEX:DEX, (2) HDEX:DEX, and (3) HDEX:HDEX. DEX increased dry adhesion more than HDEX did, which likely is due to differences in the ability to form specific interactions, especially hydrogen bonding. HDEX gave strong wet adhesion, probably due to its poorer solvency, while DEX contributed to reducing the wet adhesion due to its hydrophilicity. All combinations showed a steric repulsion on approach in aqueous media. Furthermore, when HDEX was adsorbed on either or both surfaces a long range attractive force between the surfaces was detected outside this steric regime.
\end{abstract}

(C) 2007 Elsevier Inc. All rights reserved.

Keywords: Adhesion; Interaction forces; Polymer; Adsorption; Incompatible; Asymmetric; Cationic dextran; Hydrophobic; Hydrophilic; AFM; Colloidal probe technique; JKR

\section{Introduction}

When two polymeric surfaces come into contact polymer chains from one surface can penetrate the second surface, ultimately leading to a welded joint [1]. The French polymer physics school has published extensively on theoretical aspects of mixing at the interface upon contact [2]. Welded joints are stronger because energy is consumed, pulling out the interdigitated polymer chains $[3,4]$. The fundamental requirements for interdigitation are the presence of mobile chain ends and time for interdiffusion. It was recently demonstrated that chain ends contribute to adhesion between glassy polymers [5]. Furthermore, surface force apparatus (SFA) measurements showed

\footnotetext{
* Corresponding author. Fax: +468505 51299 .

E-mail address: malin.eriksson@carmeda.se (M. Eriksson).
}

that upon approaching the glass transition temperature, adhesion increases due to entanglement formation across the interface [6]. With longer contact times, the surface molecules have more time for rearrangement and interdigitation across the opposing surfaces. Adhesion also depends on the rate of surface separation. If welded surfaces are very slowly separated, entanglements across the interface will spontaneously disengage, whereas higher separation rates will result in visco-elastic losses and chain rupture that will contribute to the measured adhesion energies [7-9]. Finally, the relative humidity and the presence of plasticizing agents during joint formation and joint separation highly influence the joint strength $[6,10]$. Solvents can promote chain mobility in brittle polymers and thereby speed up the diffusion rate.

There are numerous technologies, including surface coating with latex paints and papermaking, where adhesive joints form by removing water from suspension of surfaces coated with 
hydrophilic polymers. One example of this type of process is film formation from the coalescence of latex paint. Upon drying, the exterior polymers in the latex particles inter-diffuse to form a continuous film, a process which has been characterized by fluorescent energy transfer experiments [11]. In the case of papermaking, cellulose fibers coated with hemicellulose, starch or synthetic polymers are pressed together and the water is removed. Although the glass transition temperatures of the surface carbohydrates on dry cellulose fibers are above $200^{\circ} \mathrm{C}$ [12], the presence of plasticizing water during joint formation suggests that interdigitation of surface polymers might contribute to the remarkable adhesive strength of fiberfiber joints in paper. This was first suggested by McKenzie in 1984 [13]. McKenzie emphasizes the role of hemicelluloses as surface polymers, which mix to form bonds between the fibers. However, in most modern papermaking suspensions, the fibers are at least partially coated with an adsorbed layer of waterborne polymers added to enhance the fiber-fiber joint strength, to retain fines, fillers or pitch, and to make the fibers water repellant. Thus, the capability of these polymers to participate in interdiffusion should also be considered.

If contacting polymer surfaces are not identical, polymer compatibility is also important because, by definition, incompatible polymers do not mix. The role of compatibility was demonstrated by Iyengar and Erickson, who showed that nearly matched solubility parameters were required for development of strong adhesion between polymer films [14]. If interdigitation is important for adhesion between fibers in paper, the strength of paper should be sensitive to the compatibility of polymers in the fiber-fiber joint. This hypothesis was tested by making paper from a mixture of two separate batches of cellulose fibers-one coated with dextran and the other coated with a hydrophobically modified dextran [15]. Both dextran polymers were water soluble, however, when mixed at concentrations of $\sim 10 \%$ (w/w), the dextran polymer solutions phase separated [16], similar to the extensively studied dextran/polyethylene glycol mixtures [17] and dextran/polyethylene oxide mixtures [18]. Such aqueous biphase systems are good examples of polymer incompatibility. Thus, the hypothesis suggests that paper with many joints containing incompatible polymers in the fiber-fiber joints would be weaker [15]. The results were inconclusive, perhaps because paper is a complex and poorly defined material for fundamental adhesion studies.

In this work, an attempt is made, to access the role of polymer compatibility for development of adhesive joints between surfaces coated with hydrophilic polymers using AFM with the colloidal probe technique and continuum contact mechanics applied to the JKR (Johnsson, Kendall and Roberts) theory [19]. Specifically, three cases are compared: (1) both surfaces coated with dextran (DEX); (2) both surfaces coated with hydrophobically modified dextran (HDEX); and (3) the asymmetric case where one surface is coated with DEX and the other with HDEX. It was also anticipated that all interactions would be repulsive in water and that the asymmetric HDEX:DEX would give the weakest adhesion for both wet and dry surfaces.

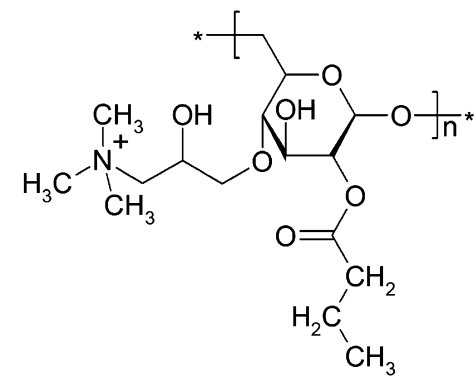

Fig. 1. A HDEX repeat unit showing the cationic and the hydrophobic modification. On average there is about one hydrophobic butyric ester on every other carbohydrate ring, whereas there are about 30 rings per quaternary ammonium group.

\section{Materials}

\subsection{Modification of dextran}

Chemicals: 3-epoxypropyl-trimethylammonium chloride (ETAC) was purchased from FLUKA. 1.4-pyrrolidinopyridine (PYP), 1,3-dicyclohexylcarbodiimide (DCC), dichloromethane and butyric acid were purchased from Aldrich. Formamide was from $\mathrm{BDH}$ and $N, N$-dimethylformamide (DMF) was from Caledon Laboratories Ltd. Dextran with a molecular weight $\left(M_{\mathrm{W}}\right)$ of $513 \mathrm{kDa}$ was purchased from Sigma.

Cationic dextrans (DEX) were prepared according to a previously described method [20,21]. In the present study, dextran was dissolved in water to a final content of $20 \%(\mathrm{w} / \mathrm{w})$ and $\mathrm{pH}$ was adjusted to $13-13.5$ with $5 \%$ (w/w) $\mathrm{NaOH}$ solution. $70 \%(\mathrm{w} / \mathrm{w})$ ETAC was added and the temperature was raised to $45^{\circ} \mathrm{C}$. After $5-6 \mathrm{~h}$, the mixture was allowed to cool at room temperature and $\mathrm{HCl}$ was added to bring the $\mathrm{pH}$ to approximately 6-7. The samples were purified using dialysis (3400$1400 M_{\mathrm{w}}$ cut-off) for at least ten days. Finally, the sample was freeze dried using a Heto Drywinner, Model DW3, and then stored at $5{ }^{\circ} \mathrm{C}$ until use.

Hydrophobically modified cationic dextran (HDEX) was prepared by esterification of the cationic dextran, using butyric acid, according to a method presented elsewhere [16,22]. In the present research, dried cationic dextran was dissolved in formamide/DMF (33/67) to get an anhydroglucose concentration of 0.65 M. Butyric acid (0.45 M) and PYP (0.025 M) were added to the reaction mixture. The mixture was stirred to form a homogeneous solution, where after DCC, dissolved in dichloromethane $50 \%$ (w/w), was added to get a DCC concentration of $0.45 \mathrm{M}$ in the reaction mixture. The reaction was performed at $30^{\circ} \mathrm{C}$ under nitrogen for $24 \mathrm{~h}$. The sample was filtrated to remove dicyclourea and then precipitated in acetone, dissolved in water, centrifuged (using a Beckman L7-55 ultracentrifuge; $30,000 \mathrm{rpm}, 25^{\circ} \mathrm{C}, 30 \mathrm{~min}$ ), dialyzed for two days, centrifuged, filtrated, and freeze dried. The products were stored at $5{ }^{\circ} \mathrm{C}$ before use. Fig. 1 presents the HDEX repeat unit showing the cationic and the hydrophobic modification.

\subsection{Substrates}

Silicon wafers (150 mm, p-type) were purchased from Memc Electronics Materials, Novara, Italy. They were washed 
consecutively with ethanol and Milli-Q water and blown dry with nitrogen. For the SPAR measurements the silicon oxide wafers were treated according to a procedure presented elsewhere to achieve a silica surface with an oxide layer of around $105 \mathrm{~nm}$, as determined by ellipsometry, using a Rudolph ellipsometer, (Model 437, Rudolph Research, Flanders NJ, USA) [23].

The mica surfaces (kindly provided by Mark Rutland, KTH, Stockholm, Sweden) were carefully cleaved several times on both sides to give a clean surface, before mounted in the AFM liquid cell.

Silica coated quartz crystals, used for the QCM-D measurements, were treated with a mixture of 3 parts sulfuric acid (VRW) and 1 part hydrogen peroxide (Merck) for $1 \mathrm{~min}$ and then rinsed with an excess of Milli-Q water and finally blown dry with nitrogen.

PDMS hemispherical caps were prepared from poly(dimethylsiloxane) (182 silicone elastomer, Dow Corning, USA) and a curing agent (182 curing agent silicone elastomer, Dow Corning, USA) according to the procedure presented elsewhere [24]. The curing agent ( 1 part by weight) was added to the silicone elastomer (10 parts by weight) under vigorous stirring. In order to remove air bubbles the mixture was treated under vacuum for $1 \mathrm{~h}$. Droplets of the reaction mixture were placed on a glass slide treated with fluorodecyltrichlorosilane (Aldrich) and then cured for $1 \mathrm{~h}$ at $105^{\circ} \mathrm{C}$, resulting in caps with a radius of approximately $1 \mathrm{~mm}$. The cured caps were extracted in heptane (WVR) for $12 \mathrm{~h}$ to remove unreacted monomers.

The film preparation of dextran onto the PDMS cap was performed according to the method outlined elsewhere [25]. Before any surface treatment, the prepared PDMS caps were oxidized in a plasma cleaner (Model PDC 002, Harrick Scientific Corporation, NY, USA; effect $7 \mathrm{~W}$ ) for $15 \mathrm{~min}$, in order to provide a surface with good adherence to the dextrans used [25]. The silicon wafers were treated in the same manner. To obtain dextran-coated silica surfaces and PDMS surfaces, they were placed in a beaker with a solution of the cationically charged dextran (hydrophobic or hydrophilic) at $\mathrm{pH} 8$, with a polymer concentration of $1 \mathrm{~g} / \mathrm{L}$, for $45 \mathrm{~min}$. Thereafter the excess polyelectrolyte was rinsed away with Milli-Q water and the surfaces were dried with a nitrogen stream. All surfaces were subsequently stored in dust-free conditions in an environment set to the relative humidity $(\mathrm{RH})$ that the measurements were performed in, i.e., $50 \%$.

\section{Methods}

\subsection{Characterization of dextrans and dextran-covered surfaces}

The degree of substitution (DS) of the modified dextrans was determined with ${ }^{1} \mathrm{H}$ NMR. A Bruker AV 200 NMR spectrometer was used to record the spectra. $\mathrm{D}_{2} \mathrm{O}$ was used as solvent and $100 \mathrm{mg}$ of dextran was dissolved in $3.5 \mathrm{ml} \mathrm{D}_{2} \mathrm{O}$. The degree of quaternary ammonium ion substitution was reported relative to the peak assigned to the anomeric proton and the degree of fatty acid substitution was reported relative to the same peak [16].
A differential refractometer was used to determine the refractive index increment $(d n / d c)$ of the dextrans used and static light scattering was used to determine $M_{\mathrm{w}}$ and the radius of gyration $\left(R_{\mathrm{g}}\right)$ with aid of a photon counting device supplied by Hamamatsu. The light source was a $3 \mathrm{~mW} \mathrm{He}-\mathrm{Ne}$ laser with a wavelength of $632.8 \mathrm{~nm}$. Stock solutions, with a dextran concentration of $2 \%(\mathrm{w} / \mathrm{w})$ in $0.1 \mathrm{M} \mathrm{NaCl}$ were prepared and dialyzed against $0.1 \mathrm{M} \mathrm{NaCl}$ solution for $24 \mathrm{~h}$. From the stock solution, five solutions in the concentration range 0.5$1.0 \%(\mathrm{w} / \mathrm{w})$ in $0.05 \mathrm{M} \mathrm{NaCl}$ were prepared and used for the $d n / d c$ determination. For the light scattering measurements, five solutions in the range $0.01-0.1 \%(\mathrm{w} / \mathrm{w})$ were used, all of which were in a background electrolyte solution of $0.05 \mathrm{M}$ $\mathrm{NaCl}$. This salt concentration is relatively low for these types of measurements; however, considering the low charge density of the dextrans this salt concentration was high enough to obtain clear Zimm plots. Prior to all measurements, the solutions were filtered using filters with a cut off size of $0.8 \mu \mathrm{m}$.

Contact angle measurements against Milli- $\mathrm{Q}$ water were performed for the silica surfaces treated with the modified dextrans using a KSV Contact angle meter, CAM 200 (Helsinki, Finland).

\subsection{Interaction studies between dextran-covered surfaces}

The micro adhesion measurement apparatus (MAMA) was used to study the dry adhesion between the dextran-covered surfaces. The equipment was constructed according to the principles described by Mangipudi et al. [26]. In this approach, an elastic cap of cross-linked poly(dimethylsiloxane) (PDMS) is pressed against a flat surface. When a predetermined maximum load is reached, unloading is initiated and performed with the same rate as the loading cycle, until the surfaces are completely separated. Since the PDMS lens is transparent the increase or decrease in contact area can be directly imaged through a microscope connected to a computer via a CCD camera. By determining the relationship between the cube of the contact radius $\left(a^{3}\right)$ and the applied load $(F)$, the work of adhesion $(W)$ between two elastic bodies can be determined by application of the Johnson, Kendall, and Roberts (JKR) theory, according to the equation [19]

$a^{3}=\frac{R}{K}\left[F+3 \pi W R+\sqrt{6 \pi W R F+(3 \pi W R)^{2}}\right]$,

where $R$, which is a function of the two contacting bodies' radii, is equal to the radius of the elastic sphere when adhesion between an elastic cap and a flat surface is analyzed. In the present work the $R$ values were about $1 \mathrm{~mm}$. The adhesion energy at minimum load $\left(W_{\min }\right)$ can be determined from the pull-off force $\left(F_{\mathrm{S}}\right)$ according to the equation

$F_{\mathrm{s}}=\frac{3}{2} \pi R W_{\min }$.

In a typical measurement, the surfaces were carefully brought into contact in such a way that a negative load was obtained. The surfaces were allowed to approach each other stepwise every $10 \mathrm{~min}$ up to a predetermined maximum load 


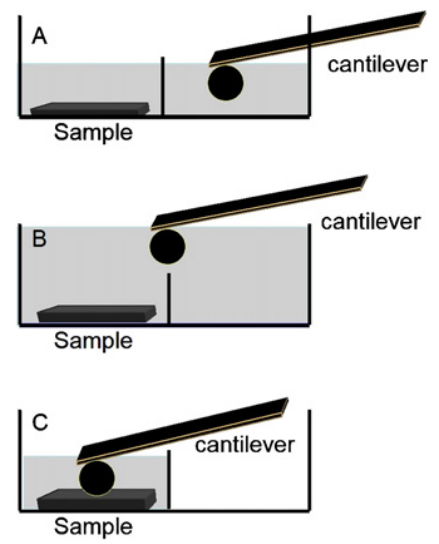

Fig. 2. Schematic representation of the liquid cell used in the AFM force measurements. After adsorption from dextran solutions of $50 \mathrm{mg} / \mathrm{L}$ in $0.01 \mathrm{M} \mathrm{NaCl}$ at $\mathrm{pH} 8$ for $60 \mathrm{~min}$ in separate sections (A) (for example, DEX on the right and HDEX on the left in the divided liquid cell) each side of the cell was rinsed with an excess of $\mathrm{NaCl}$ solution ( $0.01 \mathrm{M}$ at $\mathrm{pH}$ 8) to remove any unadsorbed DEX or HDEX. (B) The entire cell was filled with $\mathrm{NaCl}$ solution of uniform concentration and $\mathrm{pH}$, so that it overtopped the dividing wall and the probe could be moved to the left section without being exposed to the air. (C) The probe was then in the same section as the mica surface and measurement could start.

of $250 \mathrm{mg}$. Different times at the maximum load were used from 0.5 to $48 \mathrm{~h}$. Unloading was performed stepwise every $10 \mathrm{~min}$. All measurements were performed at $50 \% \mathrm{RH}$ and $23{ }^{\circ} \mathrm{C}$. A minimum of five measurements were performed for each system.

The interaction between surfaces with adsorbed layers of dextrans in an aqueous solution was studied with the AFM colloidal probe technique [27], using a Dimension 3100 SPM (Veeco Inc., Santa Barbara, USA). To measure the force between the asymmetrically covered surfaces, i.e., HDEX:DEX, a glass cell was built with a dividing wall (see Fig. 2). The flat surface and the colloidal probe were hence separated during polyelectrolyte adsorption. After adsorption, the polyelectrolyte solution on both sides was removed by flushing the cell with excess amounts of electrolyte solution. Then the entire glass cell was filled with the electrolyte solution, and the cantilever with the attached colloidal probe was moved over the dividing wall in the glass cell, without exposing the probe to air, to the section of the cell where the substrate was stored. This setup allows the adsorption of two different polymers on the colloidal probe and on the flat surface, respectively, without exposing either the probe or the flat surface to air, which would undoubtedly dry the surfaces and change the conformation of the adsorbed layer. The dextrans were adsorbed from a solution of $50 \mathrm{mg} / \mathrm{L}$ in a background electrolyte solution of $0.01 \mathrm{M}$ $\mathrm{NaCl}$ at $\mathrm{pH} 8$ on a freshly cleaved mica surface and on a silica sphere attached to the tip. In order to eliminate the effect of the substrates on adsorption, the asymmetric force measurements were performed where the dextrans was adsorbed on both the mica surface and the silica sphere. No differences in the measured interaction profiles were observed for the two different asymmetric cases. All measurements were performed using the liquid cell described above, at $\mathrm{pH} 8$, with a salt concentration of $0.01 \mathrm{M} \mathrm{NaCl}$. After adsorption of dextrans, a minimum of $1 \mathrm{~h}$ equilibration time was used before the measurement of at least 20 force curves. Representative force curves are presented in the following.

\subsection{Adsorption of dextran onto silica}

The adsorption of the modified dextrans onto silica surfaces was studied using stagnation point adsorption reflectometry (SPAR). The method and its underlying theory has been described by Dijt et al. [28]. Typically, a silica surface is mounted in the liquid cell. A polarized laser beam enters through a $45^{\circ}$ glass prism, is reflected by the silica surface, and leaves the cell through a second $45^{\circ}$ glass prism. A reflectivity ratio $(S)$ is defined from the reflectivity of the laser light in both directions of polarization. Upon adsorption of for example a macromolecule onto the surface, the refractive index of the surface layer changes, thereby changing the reflection of the incident laser beam to a certain extent. Thus, the change in intensities of the parallel and perpendicular components of the reflected laser beam $(\Delta S)$ can be determined. The theory, which is based on a four-layer (silicon, silicon oxide, polyelectrolyte, and solvent) optical model, can be used to calculate the adsorbed amount $(\Gamma)$ which is proportional to $\Delta S$, according to the equation

$\Gamma=\frac{1}{A_{\mathrm{s}}} \times \frac{\Delta S}{S}$,

where $A_{\mathrm{s}}$ is a sensitivity factor proportional to the refractive index increment $(d n / d c)$ of the studied species. $A_{\mathrm{s}}$ is also highly sensitive to the thickness of the oxide layer on the silicon wafer.

In a typical experiment, a stable base line is collected by allowing a salt solution of $0.01 \mathrm{M} \mathrm{NaCl}$ at $\mathrm{pH} 8$, flow into the cell. The silica surface is then treated with the dextrans from a solution of $50 \mathrm{mg} / \mathrm{L}$ in a background solution of $0.01 \mathrm{M} \mathrm{NaCl}$ at $\mathrm{pH}$ 8 for about $15 \mathrm{~min}$. The experiment is completed by a rinsing step with the same electrolyte concentration. Using Eq. (3) it is possible to calculate the adsorbed amount of DEX and HDEX with known $d n / d c$ and oxide layer thickness values, assuming an adsorbed polyelectrolyte thickness of $5 \mathrm{~nm}$ [28].

The adsorption of the modified dextrans was also studied using the QCM-D (quartz crystal microbalance with dissipation measurements, supplied by Q-Sense, Göteborg, Sweden). This device examines the polyelectrolyte adsorption onto a resonating, silica-coated piezoelectric quartz crystal. The adsorption onto the crystal is sensed as a decrease in the resonance frequency. If the adsorbed species is flat, uniform and rigidly attached, the change in resonance frequency is directly proportional to the added mass and can be calculated using the Sauerbrey relationship as shown in the equation [29]

$\Delta m=-\frac{C_{\mathrm{qcm}}}{n} \Delta f$,

where $C_{\mathrm{qcm}}$ is the mass sensitivity constant, $17.7 \mathrm{ng} / \mathrm{cm}^{2}, n$ is the overtone number and $\Delta f$ is the change in resonance frequency of the AT-cut quartz crystal. Deviations from the Sauerbrey relationship will sometimes, but not always, occur upon polyelectrolyte adsorption. This is due to both the conformation of adsorbed polyelectrolytes which can be adsorbed as tails and loops, and to the hydrodynamically coupled water, which 
Table 1

Characteristics of the modified dextrans used and contact angles against water for the dextran-covered surfaces

\begin{tabular}{llllllc}
\hline Polymer & $\begin{array}{l}M_{\mathrm{W}} \\
(\mathrm{kDa})\end{array}$ & $\begin{array}{l}R_{\mathrm{g}} \\
(\mathrm{nm})\end{array}$ & $\begin{array}{l}\text { Cationic } \\
\mathrm{DS}\end{array}$ & $\begin{array}{l}\text { Hydrophobic } \\
\mathrm{DS}\end{array}$ & $\begin{array}{l}d n / d c \\
(\mathrm{ml} / \mathrm{g})\end{array}$ & $\begin{array}{l}\text { Contact angle } \\
\left({ }^{\circ}\right)\end{array}$ \\
\hline DEX & 475 & 35 & 0.034 & - & 0.139 & $7 \pm 1$ \\
HDEX & 617 & 47 & 0.036 & 0.61 & 0.137 & $48 \pm 1$ \\
\hline
\end{tabular}

will also influence the decrease in resonance frequency and contribute to the detected mass uptake. The change in energy dissipation during the adsorption can also be studied, yielding information about the visco-elastic properties of the adsorbed film. To determine these properties, the electric current to the oscillating crystal is stopped so that the decay of the amplitude of the crystal can be determined. This decay is proportional to the visco-elastic properties of the layer, which are partly dependent on the amount of water trapped in the adsorbed layers. For a rigidly attached species, no change in the dissipation will be observed during adsorption. For an adsorbed visco-elastic layer (water-rich), the energy dissipated through the layer will increase during adsorption. The dissipation factor $(D)$ is defined in Eq. (5), and the change in dissipation can, according to the simplest view, be regarded as a change in the stiffness of the adsorbed layer.

$D=\frac{E_{\text {dissipated }}}{2 \pi E_{\text {stored }}}$.

In a typical experiment a stable base line is collected with a salt concentration of $0.01 \mathrm{M} \mathrm{NaCl}$ at $\mathrm{pH} 8$. The dextran solution, $50 \mathrm{mg} / \mathrm{L}$ in $0.01 \mathrm{M} \mathrm{NaCl}$ solution at $\mathrm{pH} 8$, was then injected into the liquid chamber in which the crystal is located, and allowed to adsorb for $60 \mathrm{~min}$. The rinsing solution was then injected again, and at the same time the polyelectrolyte solution was allowed to drain out. The adsorbed amount of DEX and HDEX (including the coupled water) was calculated for the third overtone using Eq. (4).

\section{Results}

\subsection{Characterization of dextrans and dextran-covered surfaces}

Dextran was rendered slightly cationic by grafting about one quaternary ammonium adduct for every 30 sugar rings. A sample of the cationic dextran (DEX) was converted to cationically hydrophobic dextran (HDEX) by grafting a butyrate ester on every other ring (see structure in Fig. 1). The composition, $M_{\mathrm{w}}$, $R_{\mathrm{g}}$, and $d n / d c$ values of DEX and HDEX are summarized in Table 1. Also shown in Table 1 are the static contact angles for water drops placed on DEX and HDEX films adsorbed onto silica and dried.

\subsection{Dry adhesion measurements between dextran-coated surfaces}

Dry adhesion measurements were performed between silica and oxidized PDMS surfaces with dextrans preadsorbed to the

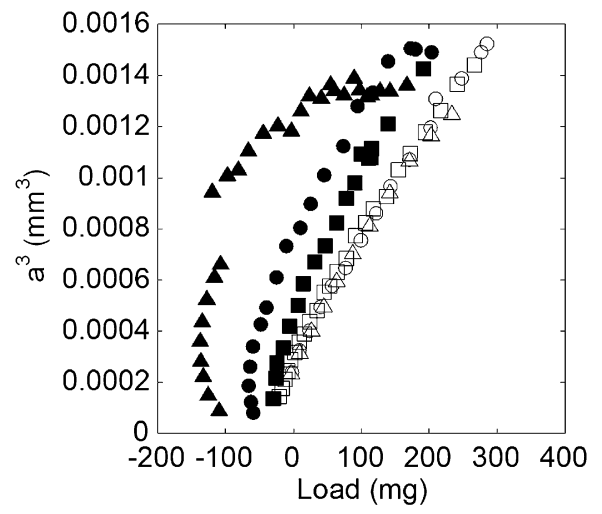

Fig. 3. Results from the adhesion measurements between dextran-covered silica surfaces and PDMS surfaces using the MAMA equipment. The adhesion was measured between combinations of DEX:DEX (triangles), HDEX:DEX (circles) and HDEX:HDEX (squares) covered surfaces. Open symbols refer to the loading data and closed symbols refer to the unloading data. The measurements were performed in air at $50 \% \mathrm{RH}$ and a temperature of $23^{\circ} \mathrm{C}$.

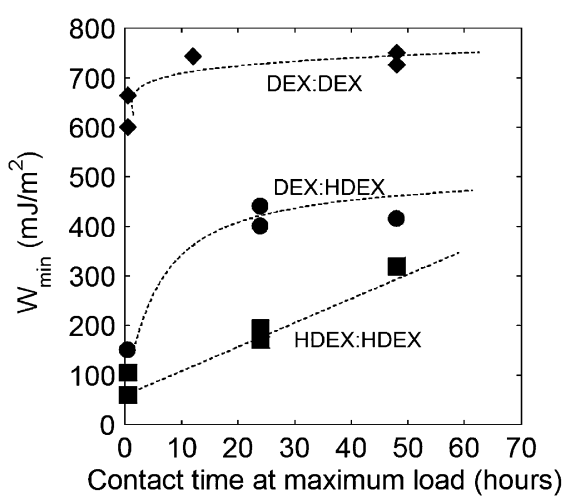

Fig. 4. The work of adhesion at minimum load $\left(W_{\min }\right)$ as a function of contact time at maximum load. The adhesion was measured between combinations of DEX:DEX (diamonds), HDEX:DEX (circles) and HDEX:HDEX (squares) covered surfaces with differences in their contact time at maximum load. The measurements were performed in air at $50 \% \mathrm{RH}$ and a temperature of $23^{\circ} \mathrm{C}$. The lines included in the figure merely serve to guide the eye.

surfaces. Three different combinations of polyelectrolytes were investigated; DEX:DEX, HDEX:HDEX, and the asymmetric case HDEX:DEX. The surfaces were loaded and unloaded in air at a controlled RH of $50 \%$ and $23{ }^{\circ} \mathrm{C}$. Fig. 3 shows results from a typical loading and unloading experiment for each system. The work of adhesion $\left(W_{\mathrm{A}}\right)$ as estimated from the loading data using Eq. (1) did not differ significantly between the three combinations, showing values in the range $40-50 \mathrm{~mJ} / \mathrm{m}^{2}$. When comparing the loading and unloading data in Fig. 3 it can be seen that all three investigated systems show adhesion hysteresis, with DEX:DEX having the largest hysteresis. The adhesion hysteresis for the asymmetric system, DEX:HDEX, as well as for HDEX:HDEX, is less pronounced but still clearly detectable. The effect of contact time at maximum load was also investigated for all three systems, showing that a longer time of contact could further increase the adhesion hysteresis. In order to quantify these effects, the work of adhesion at minimum load $\left(W_{\min }\right)$, was calculated using Eq. (2), and presented as a function of time at maximum load. These results are presented in Fig. 4, clearly showing that longer contact times 


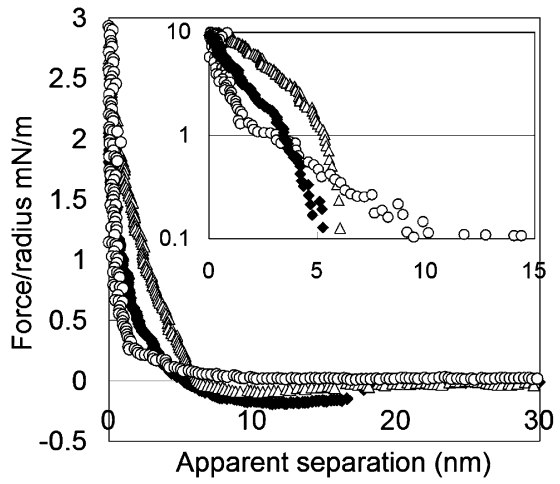

Fig. 5. Interaction forces between dextran-covered surfaces (a silica sphere, with a diameter of $10 \mu \mathrm{m}$, and a freshly cleaved mica surface) upon approach, as determined using the AFM colloidal probe technique. The dextrans were adsorbed from a solution of $50 \mathrm{mg} / \mathrm{L}$ in an electrolyte concentration of $0.01 \mathrm{M}$ $\mathrm{NaCl}$ at $\mathrm{pH}$ 8. The force measurements were performed in the same background electrolyte concentration and $\mathrm{pH}$. Open circles refer to the interaction between DEX:DEX, open triangles refer to HDEX:HDEX and closed diamonds refer to HDEX:DEX. The insert shows the same data on a logarithmic scale.

at maximum load increases the value of $W_{\min }$ for all combinations. Furthermore, Fig. 4 indicates that DEX:DEX and HDEX:DEX reach a maximum adhesion force, after contact times longer than around $12 \mathrm{~h}$, whereas HDEX:HDEX did not reach a maximum within the time frame of the present investigation.

\subsection{Interactions between dextran-coated surfaces in aqueous solution}

The interaction between a dextran-covered colloidal probe (silica sphere) and a dextran-covered mica surface was determined using colloidal probe AFM. In accordance with the measurements performed under ambient conditions, the same three combinations of polyelectrolytes were investigated; DEX:DEX, HDEX:HDEX, and the asymmetric case HDEX:DEX. By using the specially developed measuring chamber, shown in Fig. 2, it was possible to measure the interaction between both symmetrically and asymmetrically covered surfaces with no polymer in solution and without exposure to air. The results from these measurements are shown in Fig. 5 (approach) and Fig. 6 (separation).

Fig. 5 show that all three cases display a steric interaction profile, which is typical for interactions between polymercovered surfaces. Hence, there is an indication that both types of dextrans are adsorbed with a somewhat extended conformation, as would be expected for a polyelectrolyte with a low charge density. The HDEX:HDEX combination, results in a larger repulsive force, as compared with the HDEX:DEX and DEX:DEX combinations, when the surfaces are brought together. At surface separations outside the steric region, a weak attractive force is observed for cases involving HDEX on either or both surfaces up to separations of $20 \mathrm{~nm}$.

As shown in Fig. 6, the adhesion force is almost nondetectable between two DEX surfaces, while there is considerable adhesion between two HDEX surfaces. The HDEX:DEX case shows an intermediate adhesion force.

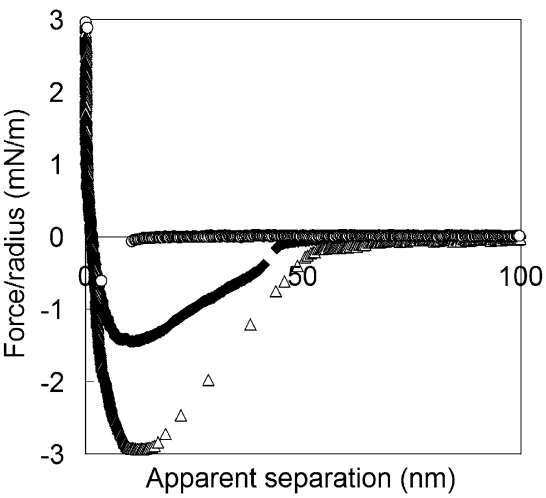

Fig. 6. Interaction forces between dextran-covered surfaces (a silica sphere, with a diameter of $10 \mu \mathrm{m}$, and a freshly cleaved mica surface) on separation as determined using the AFM colloidal probe technique. The dextrans were adsorbed from a solution of $50 \mathrm{mg} / \mathrm{L}$ in an electrolyte concentration of $0.01 \mathrm{M}$ $\mathrm{NaCl}$ at $\mathrm{pH}$ 8. The force measurements were performed in the same background electrolyte concentration and $\mathrm{pH}$. Open circles refer to the interaction between DEX:DEX, open triangles refer to HDEX:HDEX and closed diamonds refer to HDEX:DEX.

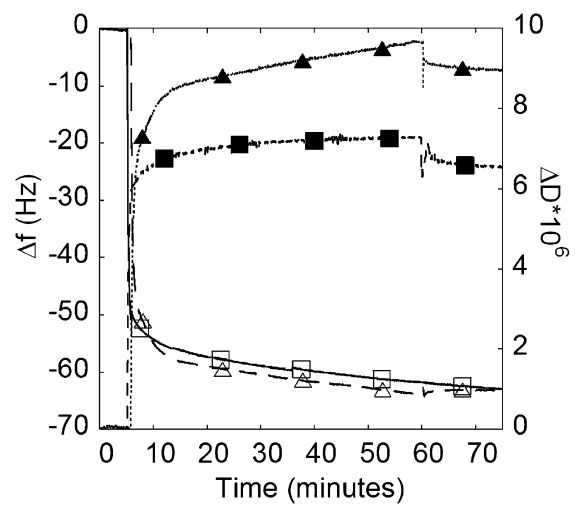

Fig. 7. Adsorption of DEX (triangles) and HDEX (squares) from a solution of $50 \mathrm{mg} / \mathrm{L}$ dextran in $0.01 \mathrm{M} \mathrm{NaCl}$ at $\mathrm{pH}$ 8, using QCM-D. The two lower curves with open symbols represent the change in frequency upon adsorption onto the oscillating silica-coated quartz crystal. The two upper curves with closed symbols represent the change in dissipation due to adsorption of DEX and HDEX. The initial baseline and the final rinsing step were performed with a $\mathrm{NaCl}$ solution of $0.01 \mathrm{M}$ at $\mathrm{pH} 8$.

\subsection{Adsorption of dextran onto silica}

In order to obtain more information regarding the wet interaction measurements, the adsorption of DEX and HDEX was studied using the quartz crystal microbalance with dissipation measurements (QCM-D) and stagnation point adsorption reflectometry (SPAR). Data from the QCM-D measurements are presented in Fig. 7, showing the change in frequency (third overtone) and dissipation upon adsorption of DEX and HDEX onto silica. The change in frequency was essentially the same regardless of the type of dextran used. Interestingly, significant differences in the dissipation values were obtained and the adsorption of DEX resulted in the higher dissipation value. Fig. 7 also shows that most of the dextran is adsorbed within a few minutes whereafter a plateau is slowly reached. After $60 \mathrm{~min}$, when the excess polyelectrolyte is rinsed out with the electrolyte solution, there is a decrease in dissipation for 


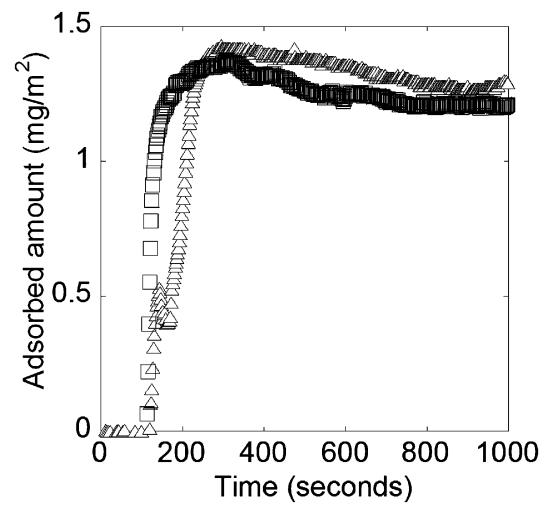

Fig. 8. Adsorbed amounts of DEX (triangles) and HDEX (squares) onto a silica surface, calculated from SPAR data using Eq. (1). The adsorptions were made from a solution of $50 \mathrm{mg} / \mathrm{L}$ dextran in $0.01 \mathrm{M} \mathrm{NaCl}$ at $\mathrm{pH}$. The initial baseline $(0-100 \mathrm{~s})$ and the final rinsing step (900-1000 s) were performed with a $\mathrm{NaCl}$ solution of $0.01 \mathrm{M}$ at $\mathrm{pH} 8$.

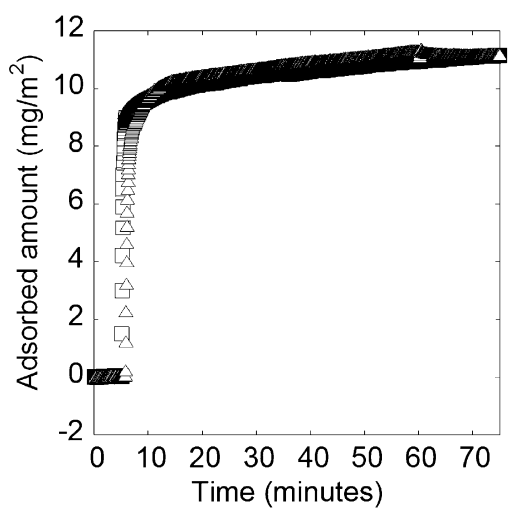

Fig. 9. Adsorbed amounts of DEX (triangles) and HDEX (squares) onto a silica surface, calculated from QCM-D data using Eq. (2). The adsorptions were made from a solution of $50 \mathrm{mg} / \mathrm{L}$ dextran in $0.01 \mathrm{M} \mathrm{NaCl}$ at $\mathrm{pH}$ 8. The initial baseline and the final rinsing step were performed with a $\mathrm{NaCl}$ solution of 0.01 at $\mathrm{pH} 8$.

both DEX and HDEX while the frequency remains unchanged. The adsorbed amount of dextran was calculated from the data collected from the SPAR measurement using Eq. (3), and is presented as a function of time in Fig. 8. The adsorption was performed in the same manner as for the QCM-D measurements, but the adsorption time was only monitored for about 15 min since a plateau value was readily obtained after this time, indicating equilibrium in adsorbed amount. There was no significant difference between DEX and HDEX regarding the calculated adsorbed amount, approximately $1.25 \mathrm{mg} / \mathrm{m}^{2}$ in both cases. From the QCM-D data, the adsorbed amount of the dextrans, including the hydrodynamically coupled water could be calculated using Eq. (4). The results are presented in Fig. 9, showing values of about $11 \mathrm{mg} / \mathrm{m}^{2}$ for both DEX and HDEX (after the rinsing step). From Figs. 8 and 9 it can be concluded that the adsorbed structures of DEX and HDEX contain approximately $90 \%$ water. These values are also consistent with the measured dissipation values for both DEX and HDEX, which were quite high, as shown in Fig. 7. However, since the adsorbed layers contain the same amount of coupled water this does not fully explain the detected differences in dissipation.

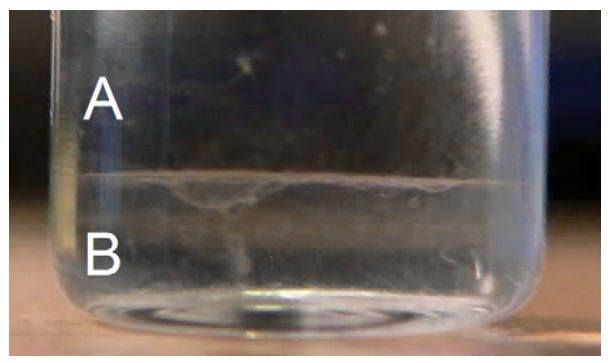

Fig. 10. Biaqueous phase separation of DEX and HDEX in 10\% (w/w) concentration in $0.01 \mathrm{M} \mathrm{NaCl}$. These conditions were chosen since the QCM-D and SPAR data indicated that the amount water in an adsorbed dextran layer were about $90 \%$, both for DEX and HDEX. The upper phase (A) is rich in HDEX and the lower phase $\mathrm{B}$ is rich in DEX.

\section{Discussion}

Both DEX and HDEX are water soluble. However, mixtures of the two polymers exhibit aqueous biphase separation. The phase diagram for similar mixtures has been published elsewhere [16]. Fig. 10 shows a photograph of a $10 \%$ (w/w) mixture of the dextrans used in this work. Thus, it was anticipated that incompatibility of the polymer solutions would be reflected in the interactions of adsorbed layers. In other words, our hypothesis was that the DEX/HDEX combination would give the lowest adhesion. This was not the case.

Force measurements, performed in aqueous solution, using colloidal probe AFM displayed a repulsive force on approach for all combinations of HDEX- and DEX-coated surfaces, as indicated in Fig. 5. We propose that this is due to steric interactions similar to many examples in Refs. [31,35,36]. A closer lock into the AFM data on approach also reveals some sort of relaxation in the hard-wall zone for the DEX:DEX and HDEX:DEX cases. This is possible due to disentanglement or stretching of bridging segments of the polymer molecues. The exact origin is not possible to determine using this technique. Another interesting feature found in Fig. 5 is the attractive force at separations outside the steric regime in the HDEX:DEX and HDEX:HDEX systems. There are previous studies where this long-range attractive force has been observed between a hydrophobic and a hydrophilic surface, as well as between two hydrophobic surfaces [37-40] and the explanation remains under debate [41]. In our case neither surface is hydrophobic; the HDEX covered surface has the lower surface energy with a water contact angle of $48^{\circ}$. In summary, although the long range attractive force is repeatable, we do not understand its origin.

Upon separation, all three combinations (HDEX:DEX, DEX:DEX and HDEX:HDEX) were adhesive, as indicated by the measured pull off forces (Fig. 6). It was hypothesized that the incompatible pair (HDEX:DEX) would give the lowest adhesion reflecting the absence of interpenetration in contacting surfaces. However, this was not the case. The order of the pull off force was HDEX:HDEX > HDEX:DEX > DEX:DEX. Therefore, there was no indication from the wet adhesion results that polymer compatibility played a role.

These results can possibly be explained by the structures of the adsorbed polymer layers. From the adsorption data, us- 
ing QCM-D and SPAR, the polymer concentration within the adsorbed layer of DEX and HDEX on silica was estimated to be about $10 \%$ (same concentrations showed phase separation in solution), which shows good agreement with previous investigations [30-34]. However, it must be stressed that the concentration profile inside the two swollen dextran layers is not known; we presume that dextran concentration decreases away from the surface. Hence a much lower concentration in the outer parts of the adsorbed layers could explain the absence of the phase separation in the present interaction study. Furthermore, HDEX showed lower energy dissipation through the adsorbed layer than DEX did. This suggests that the adsorbed HDEX layer was stiffer. The amount of entrained water within the polymer layers will largely be determined by the hydrodynamic thickness of the adsorbed polymers away from the interface and not the bound water molecules to the adsorbed polymeric chains. Thus, only a relatively few polymer segments extending significantly away from the solid-liquid interface will shift the plane of shear sufficiently such that a high proportion of solvent molecules will be sensed using the QCM$\mathrm{D}$ technique. The dissipation data will be dominated by events (such as hydrophobic association) very near the interface where the polymer concentration is expected to be relatively high and only a few associative links between the adsorbed chains will probably be enough to change the stiffness of the adsorbed layer.

Another possible explanation to the detected results could be the solvency behavior of the polyelectrolytes. The HDEX: HDEX shows the strongest adhesion force on separation, which could be due to a rearrangement of the hydrophobic moieties on the modified dextran in which the hydrocarbon chains on opposite surfaces associate. This would result in a higher adhesion force as the surfaces are separated and the hydrophobic hydrocarbon chains are pulled into a poorer solvent, which is less favorable entropically compared to staying in contact with each other. The adhesion force, which is detectable at a much larger separation than the force on approach, also indicates an extension of the interacting polymer layers. This extension can not be seen in the DEX:DEX interaction. Instead a clear snap off is seen as the surfaces are separated, most likely originating from the hydrophilic nature of the polyelectrolyte. High water solubility leads to a much lower adhesion force upon separation. The intermediate adhesion force detected for the HDEX:DEX system is suggested to be due to a preferred mixing of HDEX with DEX instead of being surrounded by the solvent molecules. The strong adhesion between HDEX:HDEX and HDEX:DEX could also be a result of some sort of entanglement between the adsorbed polyelectrolytes.

The dry $(50 \% \mathrm{RH})$ adhesion energies, estimated from pull off forces (Fig. 4), showed the opposite trend than did the wet adhesion measurements (i.e., DEX:DEX > HDEX:DEX > HDEX:HDEX). Similar to the wet adhesion measurements, there is no evidence of incompatibility playing a role since the adhesion between the asymmetric surfaces was intermediate. It is generally accepted that carbohydrate surfaces, such as cellulose, show remarkably high adhesion because of hydrogen bonding, if the surfaces can achieve molecular contact in the absence of water. These effects on adhesion hysteresis and the presence of $\mathrm{OH}$ groups have been studied previously, showing a decrease in adhesion hysteresis with removal of $\mathrm{OH}$ groups from the surface $[42,43]$. It seems reasonable to suggest that the presence of butyrate groups on HDEX interfere with hydrogen bonding, resulting in lower adhesion hysteresis, see Fig. 3. This is further supported by the contact angle measurements, clearly showing that the DEX surface is more hydrophilic than the HDEX surface. The higher contact angle for the HDEX surface can be attributed to the removal of the polar $\mathrm{OH}$ groups and the introduction of the hydrophobic moieties.

The HDEX:HDEX and HDEX:DEX dry adhesion did increase with contact time over $50 \mathrm{~h}$ (Fig. 4). This could be an indication that interdiffusion across the joint is important. This type of polymer/segmental migration has been found to be relevant for the development of adhesion and more importantly, adhesion hysteresis $[6,9,10]$. For other systems, adhesion between surfaces has been found to be both time and temperature dependent [6].

\section{Conclusions}

Wet and dry adhesive interactions between surfaces bearing adsorbed dextran (DEX) or hydrophobically modified dextran (HDEX) were measured to test the hypothesis that incompatible polymer coatings give lower adhesion when forced together. The main conclusions are:

(1) For both wet and dry measurements, adhesion between DEX and HDEX coated surfaces was intermediate to the behavior of DEX:DEX and HDEX:HDEX surfaces. Therefore, the incompatibility of HDEX:DEX mixtures in solution was not reflected in wet or dry joint strength.

(2) Grafting short (butyrate ester) chains onto dextran, to convert DEX to HDEX, influenced both wet and dry adhesion but in opposite ways - wet adhesion was increased and dry adhesion decreased.

(3) Based on the results it is suggested that dry adhesion is dominated by hydrogen bonding between contacting carbohydrates and that the butyrate ester groups in HDEX interfere with hydrogen bonding.

(4) The higher wet adhesion between HDEX coated surfaces may reflect the solvency behavior of the dextran, as the hydrophobic moieties on the modified dextran rather stay in contact than being pulled out into the poorer solvent.

\section{Acknowledgments}

Malin Eriksson acknowledges KTH, Royal Institute of Technology, and Lyckeby Stärkelsen Research Foundation for financial support. Shannon M. Notley would like to acknowledge financial support from the Co-operative Research Centre for Functional Communication Surfaces. Göran Svensk, Uppsala University, and Oskar Werner, KTH, are thanked for their assistance with light scattering and contact angle measurements, respectively. 


\section{References}

[1] S.S. Voiutskii, Autohesion and Adhesion of High Polymers, Wiley, New York, 1963.

[2] F. Brochard-Wyart, P.G. de Gennes, L. Leger, Y. Marciano, E. Raphael, J. Phys. Chem. 98 (1994) 9405.

[3] E. Raphael, P.G. De Gennes, J. Phys. Chem. 96 (1992) 4002.

[4] H. Ji, P.G. De Gennes, Macromolecules 26 (1993) 520.

[5] N. Chen, N. Maeda, M. Tirrell, J. Israelachvili, Macromolecules 38 (2005) 3491.

[6] G. Luengo, J. Pan, M. Heuberger, J.N. Israelachvili, Langmuir 14 (1998) 3873.

[7] Y.L. Chen, C.A. Helm, J.N. Israelachvili, J. Phys. Chem. 95 (1991) 10736.

[8] Y.L. Chen, C.A. Helm, J.N. Israelachvili, Langmuir 7 (1991) 2694.

[9] S. Perutz, E.J. Kramer, J. Baney, C.Y. Hui, C. Cohen, J. Polym. Sci. B Polym. Phys. 36 (1998) 2129.

[10] D. Leckband, Y.L. Chen, J. Israelachvili, H.H. Wickman, M. Fletcher, R. Zimmerman, Biotechnol. Bioeng. 42 (1993) 167.

[11] J. Feng, M.A. Winnik, Macromolecules 30 (1997) 4324.

[12] A. Bristow, P. Kolseth, Paper Structure and Properties, Dekker, New York, 1986.

[13] A.W. McKenzie, Appita 37 (1984) 580.

[14] Y. Iyengar, D.E. Erickson, J. Appl. Polym. Sci. 2 (1967) 2311.

[15] R. Pelton, J. Zhang, L. Wågberg, M. Rundlöf, Nordic Pulp Paper Res. J. 15 (2000) 400.

[16] J. Zhang, R. Pelton, L. Wågberg, Colloid Polym Sci. 276 (1998) 476.

[17] L. Piculell, S. Nilsson, L. Falck, F. Tjerneld, Polym. Commun. 32 (1991) 158.

[18] M.W. Edelman, E. Van Der Linden, R.H. Tromp, Macromolecules 36 (2003) 7783.

[19] K.L. Johnson, K. Kendall, A.D. Roberts, Proc. R. Soc. London A 324 (1971) 301.

[20] J. Zhang, R. Pelton, L. Wågberg, M. Rundlöf, J. Pulp Paper Sci. 27 (2001) 145.

[21] O.B. Wurzburg, Editor Modified Starches, Properties and Uses, 1987.
[22] C.H. Bamford, I.P. Middleton, K.G. Al-Lamee, Polymer. 27 (1986) 1981.

[23] M. Eriksson, S.M. Notley, L. Wågberg, J. Colloid Interface Sci. 292 (2005) 38.

[24] J. Forsström, M. Eriksson, L. Wågberg, J. Adhes. Sci. Technol. 19 (2005) 783.

[25] M. Tirrell, Langmuir 12 (1996) 4548.

[26] V.S. Mangipudi, E. Huang, M. Tirrell, A.V. Pocius, Macromolecular Symposia. 1996, p. 102 (9th Rolduc Polymer Meeting, Smart Polymer Materials \& Products, 1995, p. 131).

[27] W.A. Ducker, T.J. Senden, R.M. Pashley, Nature 353 (1991) 239.

[28] J.C. Dijt, M.A. Cohen Stuart, J.E. Hofman, G.J. Fleer, Colloids Surf. 51 (1990) 141.

[29] M. Rodahl, F. Höök, A. Krozer, P. Brzezinski, B. Kasemo, Rev. Sci. Instrum. 66 (1995) 3924.

[30] F. Höök, B. Kasemo, T. Nylander, C. Fant, K. Sott, H. Elwing, Anal. Chem. 73 (2001) 5796.

[31] S.M. Notley, S. Biggs, V.S.J. Craig, L. Wågberg, Phys. Chem. Chem. Phys. 6 (2004) 2379.

[32] V.S.J. Craig, M. Plunkett, J. Colloid Interface Sci. 262 (2003) 126.

[33] S.M. Notley, M. Eriksson, L. Wågberg, J. Colloid Interface Sci. 292 (2005) 29.

[34] M.A. Plunkett, P.M. Claesson, M. Ernstsson, M.W. Rutland, Langmuir 19 (2003) 4673.

[35] S. Biggs, Langmuir 11 (1995) 156.

[36] S.M. Notley, V.S.J. Craig, S. Biggs, Macromolecules 36 (2003) 2903.

[37] Y.H. Tsao, D.F. Evans, H. Wennerström, Langmuir 9 (1993) 779.

[38] Y.H. Tsao, D.F. Evans, H. Wennerström, Science 262 (1993) 547.

[39] Y.H. Tsao, S.X. Yang, D.F. Evans, H. Wennerström, Langmuir 7 (1991) 3154.

[40] J.L. Parker, P.M. Claesson, Langmuir 10 (1994) 635.

[41] H.K. Christenson, P.M. Claesson, Adv. Colloid Interface Sci. 91 (2001) 391.

[42] S. Kim, G.Y. Choi, A. Ulman, C. Fleischer, Langmuir 13 (1997) 6850.

[43] M. Rundlöf, M. Karlsson, L. Wågberg, E. Poptoshev, M. Rutland, P. Claesson, J. Colloid Interface Sci. 230 (2000) 441. 